\title{
Relationship Between Brain Hemodynamic State, Autonomic Nerve Input, and Symptoms of Atrial Fibrillation
}

\author{
Takumi J Matsubara, ${ }^{1}$ MD and Katsuhito Fujiu, ${ }^{1,2}$ MD
}

(Int Heart J 2019; 60: 1233-1235)

$\mathrm{T}$ he number of patients with atrial fibrillation (AF) is increasing worldwide. Typical symptoms of AF are varied. The most common symptoms include palpitations, shortness of breath, fatigue, and anxiety, while the most deleterious symptom is cerebral infarction. $^{1-3)}$ These symptoms severely impair a patient's quality of life (QOL), and the goal of medical treatment for AF is not only improvement of the patient's prognosis, but also QOL.

\section{Article p.1328}

As QOL depends on the subjective feelings or sensations of the patient during AF, it is difficult to assess each patient's QOL objectively. Objective assessment of the symptoms is necessary to treat patients properly. Kato, et $a l^{4)}$ described the efficacy of pulmonary vein isolation (PVI) on patient QOL. In their report, the authors assessed the QOLs using a mental component summary (MCS) and physical component summary (PCS). MCS and PCS are parameters of medical outcomes study in the 36-item short-form health survey (SF-36). SF-36 has been regarded as a general health-based survey of QOL. ${ }^{5,6)}$ The main components of MCS include mental health, vitality, and emotional role. On the other hand, PCS mainly consists of physical function, bodily pain, and general health $^{4)}$. A lower score in MCS or PCS means a low QOL.

According to the present study from Kato, et al, improvement of QOL after PVI in AF patients was observed for both paroxysmal $\mathrm{AF}$ (PAF) and persistent $\mathrm{AF}(\mathrm{PeAF}){ }^{4)}$ One of the interesting points was that the improvement of QOL differed between PAF and PeAF patients. The QOL of PAF patients improved MCS than that of PCS. PeAF patients had much better improvement in PCS than MCS. The decrease in MCS was assumed to be due to improvements in symptoms such as palpitations, chest discomfort, and anxiety for arrhythmia attack. The alteration of heart rhythm is one of the causes of AF symptoms including palpitations and chest discomfort. Since the prediction of an $\mathrm{AF}$ attack is impossible, the patient might have con- tinuous anxiety concerning an AF attack which leads them to suffer mental stress or poor vitality.

Improvement of PCS was also observed in PAF patients; the degree of improvement was not greater than in PeAF patients. This phenomenon could be explained with insight into the difference in AF duration. PAF patients have a shorter duration of AF events. As PAF occurs intermittently, the patients experience sudden onset of the symptoms in short duration but most of the time, within no AF attack duration, patients'exercise tolerance is preserved.On contrary, in PeAF patients, symptoms such as palpitations and chest discomfort disappear gradually. Finally, as shown in the present study, almost all patients with PeAF become asymptomatic. The disappearance of symptoms due to lasting of AF could be the reason that post PVI improvement of MCS was limited in PeAF patients compared to that of PAF patients.

Interestingly, the improvement of PCS in PeAF patients was greater than in PAF patients. In PeAF patients, even asymptomatic, physical activity such as exercise tolerance was reduced. Recovery of physical activity by PVI might contribute to the improvement of PCS in PeAF. The results of the present study seem to support the benefit of PVI on PeAF patients.

Not only the occurrence of the symptoms, but also the degree of reduction of QOL was different according to the type of AF. It has been assumed that the symptoms were caused by hemodynamic changes during AF, altering left ventricular filling pressure and reducing cardiac output caused by atrioventricular dyssynchrony. ${ }^{1,8)}$ Reduced cardiac output causes abnormal perfusion of the organs which induces impaired function of each organ such as renal dysfunction, heart failure and cognitive dysfunction. ${ }^{7,8)}$ A relationship between $\mathrm{AF}$ and heart failure has been reported, as well as between $\mathrm{AF}$ and dementia. It also has been reported that persistent $\mathrm{AF}$ provokes brain atrophy and dementia via malperfusion of the brain. ${ }^{9-11)}$ The atrophic sites of the brain include the frontal lobe, temporal lobe, hypothalamus, hippocampus and amygdala. ${ }^{12)}$ These areas, especially the hypothalamus and amygdala, are associated with the autonomic nervous system in the brain. ${ }^{12)}$

From the ${ }^{1}$ Department of Cardiovascular Medicine, Graduate School of Medicine, The University of Tokyo, Tokyo, Japan and ${ }^{2}$ Department of Advanced Cardiology, Graduate School of Medicine, The University of Tokyo, Tokyo, Japan.

Address for correspondence: Takumi Matsubara, MD or Katsuhito Fujiu, MD, Department of Cardiovascular Medicine, Graduate School of Medicine, The University of Tokyo, 7-3-1 Hongo, Bunkyo-ku, Tokyo, 113-8655 Japan. E-mail: medical.natureboy@gmail.com or fujiu-tky@umin.ac.jp

Received for publication October 20, 2019. Revised and accepted October 21, 2019.

doi: 10.1536/ihj.19-563

All rights reserved by the International Heart Journal Association. 


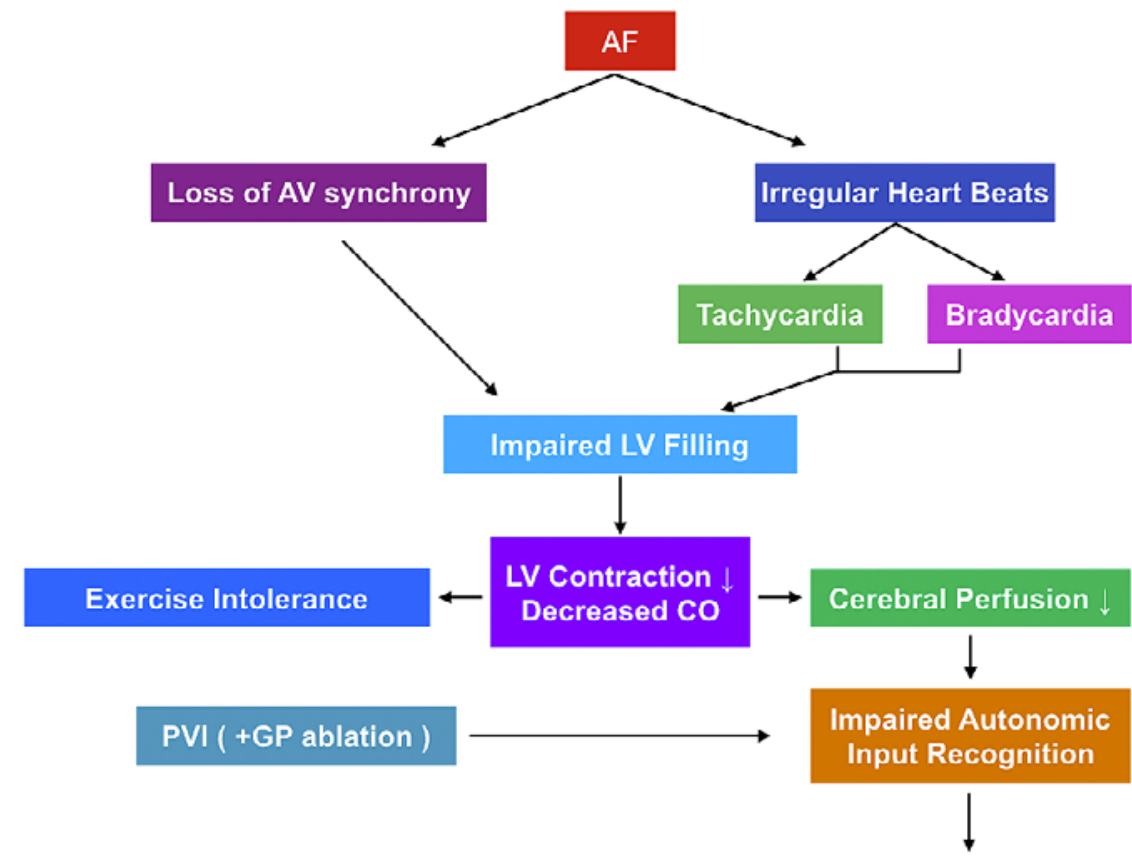

Debilitating Symptoms

Figure. Presumed mechanism of the disappearance of AF symptoms. Once AF has occurred, irregular heart beats and AV dyssynchrony impair LV filling which leads to reduction of LV contraction and cardiac output. Reduced cardiac output provokes exercise intolerance and cerebral perfusion reduction. Malperfusion of the brain, especially an autonomic center such as the hypothalamus, causes impaired recognition of autonomic inputs from the heart, and finally the AF symptoms disappear. Catheter ablation, especially ganglia plexus ablation, diminishes autonomic input from the heart to some extent. Thus, the $\mathrm{AF}$ symptoms gradually disappear. Reduced autonomic input by catheter ablation also debilitates $\mathrm{AF}$ symptoms, which leads to AF being asymptomatic even in recurrence in previously symptomatic patients. AV indicates atrioventricular; $\mathrm{CO}$, cardiac output; LV, left ventricle; and GP, ganglionated plexus.

Not only brain dysfunction, but also impaired autonomic input is one of the causes of a decrease in AF symptoms such as palpitations as observed in patients with diabetes mellitus or post ablated patients (Figure). ${ }^{13,14)}$ These reports hypothesize that inputs of the autonomic nervous system and center of the autonomic nervous system are one of the pathways of information from the heart. ${ }^{15)}$ Cardiac rhythm and contraction are regulated by the autonomic nervous system via its efferent fibers, and afferent fibers are also present in the autonomic nerves which transfer cardiac information such as myocardial ischemia during a myocardial infarction. ${ }^{16,17)}$ It seems to be reasonable to infer that successively transferred inputs from the heart during persistent AF by the autonomic nervous system to the brain provoke disappearance of the symptoms such as palpitations or chest discomfort. ${ }^{15)}$ Absence of such symptoms may relieve the patient's anxiety and ultimately mask the reduced mental QOL. On the contrary, exercise intolerance is caused by transient hemodynamic incompetence ${ }^{15)}$ therefore, symptoms such as dyspnea on effort remained in apparently asymptomatic patients, which leads to decreased physical QOL.

As general cognitive dysfunction is regarded as an advanced symptom of malperfusion of the brain, ${ }^{10)}$ an early stage of the deleterious effect of persistent AF might be "disappearance of the symptoms" due to dysfunction of specific sites of the brain such as the frontal lobe or temporal lobe. The impaired recognition of the symptoms by the autonomic nerve inputs from the heart could be one of the reasons for the poor improvement of mental QOL after PVI in asymptomatic PeAF patients. Once the AF was terminated and sinus rhythm returned, the hemodynamic state also returns to the physiological range gradually and improves exercise tolerance in PeAF patients. During exercise, not only resting state, but also a regular heart beat and proper heart rate enable the supply of sufficient cardiac output which leads to greater improvement of physical QOL in PeAF patients.

The results of the report by Kato, et al showed the benefit of PVI in PeAF patients and not only PAF patients. ${ }^{4)}$ In the clinical guideline from the European Society of Cardiology, persistent atrial fibrillation without symptoms is not a target of catheter ablation. Considering the report, sinus rhythm recovery from PeAF by catheter ablation, even without symptoms, seemed to have therapeutic potential that was greater than we expected. 


\section{Disclosure}

Conflicts of interest: The authors declare that they do not have any conflicts of interest related to this study.

\section{References}

1. Rienstra M, Lubitz SA, Mahida S, et al. Contemporary reviews in cardiovascular medicine symptoms and functional status of patients with atrial fibrillation. Circulation 2012; 125: 2933-43.

2. Dilaveris PE, Kennedy HL. Silent atrial fibrillation: epidemiology, diagnosis, and clinical impact. Clin Cardiol 2017; 40: 4138.

3. Sanna T, Diener HC, Passman RS, et al. Cryptogenic stroke and underlying atrial fibrillation. N Engl J Med 2014; 370: 2478-86.

4. Kato M, Miake J, Ogura K, et al. Different effects of pulmonary vein isolation on quality of life between patients with persistent and paroxysmal atrial fibrillation. Int Heart J 2019; 60: 132833.

5. Lins L, Carvalho FM. SF-36 total score as a single measure of health-related quality of life: Scoping review. SAGE Open Medicine 2016; 4: 2050312116671725.

6. Farivar SS, Cunningham WE, Hays RD. Correlated physical and mental health summary scores for the SF-36 and SF-12 Health Survey, V.1. Health Qual Life Outcomes 2007; 5: 350-8.

7. Böhm M, Ezekowitz MD, Connolly SJ, et al. Changes in renal function in patients with atrial fibrillation: An analysis from the RE-LY trial. J Am Coll Cardiol 2015; 65: 2481-93.

8. de Bruijn RF, Heeringa J, Wolters FJ, et al. Association between atrial fibrillation and dementia in the general population. JAMA Neurol 2015; 72: 1288-7.

9. Piers RJ, Nishtala A, Preis SR, et al. Association between atrial fibrillation and volumetric magnetic resonance imaging brain measures: Framingham Offspring Study. Heart Rhythm 2016; 13: 2020-4.

10. Berman JP, Norby FL, Mosley T, et al. Atrial fibrillation and brain magnetic resonance imaging abnormalities. Stroke 2 019; 50: 783-8.

11. Gardarsdottir M, Sigurdsson S, Aspelund T, et al. Atrial fibrillation is associated with decreased total cerebral blood flow and brain perfusion. Europace 2017; 20: 1252-8.

12. Tahsili-Fahadan P, Geocadin RG. Heart-Brain Axis Effects of Neurologic Injury on Cardiovascular Function. Circulation Research 2017; 120: 559-72.

13. Sugishita K, Shiono E, Sugiyama T, Ashida T. Diabetes influences the cardiac symptoms related to atrial fibrillation. Circ J 2003; 67: 835-8

14. Verma A, Champagne J, Sapp J, et al. Discerning the incidence of symptomatic and asymptomatic episodes of atrial fibrillation before and after catheter ablation (DISCERN AF). JAMA Intern Med 2013; 173: 149-8.

15. MacRae CA. Symptoms in atrial fibrillation: why keep score? Circ Arrhythm Electrophysiol 2009; 2: 215-7.

16. Brown AM. Exitation of afferent cardiac sympathetic nerve fibers during myocardial ischemia. J Physiol 1967; 190: 35-53.

17. Malliani A, Recordati G, Schwartz PJ. Nervous activity of afferent cardiac sympathetic fibers with atrial and ventricular endings. J Physiol 1973; 229: 457-69. 